\title{
Neurorrafia hemiipoglosso-facial após dissecção intratemporal do nervo facial: análise retrospectiva de 13 pacientes
}

\author{
Roberto S. Martins ${ }^{1,2}$, Mario G. Siqueira ${ }^{1}$ \\ Disciplina de Neurocirurgia do Hospital das Clínicas da Faculdade de Medicina da Universidade de São Paulo - São Paulo, SP, Brasil \\ Serviço de Neurocirurgia do Hospital Santa Marcelina - São Paulo, SP, Brasil
}

\section{RESUMO}

Introdução: Apesar de a neurorrafia hipoglosso-facial ser considerada uma técnica efetiva para o tratamento da paralisia facial após uma lesão proximal do nervo facial, a freqüente atrofia da hemilíngua no pós-operatório conseqüente à secção completa do nervo hipoglosso é uma complicação muitas vezes incapacitante. Uma das técnicas utilizadas para evitar essa complicação é a neurorrafia hemiipoglossofacial. Nessa técnica, o nervo facial é dissecado no seu canal, seccionado, deslocado caudalmente e suturado com metade do nervo hipoglosso na região cervical. Objetivo: Analisar retrospectivamente os resultados da técnica de neurorrafia hemiipoglosso-facial em 13 pacientes. Método: Os resultados foram avaliados por meio da classificação da paralisia facial na escala de House-Brackmann. Resultados: A idade média dos pacientes foi de 42,2 anos e a cirurgia foi realizada no período médio de 6,8 meses após a lesão do nervo facial. Em todos os pacientes, a paralisia era completa. A maioria dos pacientes (70\%) apresentou melhora significativa da paralisia facial, de completa para graus II e III. Nenhum paciente apresentou sintomas relacionados à disfunção da hemilíngua. Uma correlação positiva foi identificada entre a idade e o resultado $(p<0,05)$ e entre o tempo da lesão até a cirurgia e o resultado final $(p<0,05)$. Conclusões: Concluímos que a neurorrafia hemiipoglosso-facial apresenta bons resultados funcionais, sem complicações significativas. Recomenda-se que a cirurgia seja realizada em um menor tempo possível após a lesão confirmada do nervo facial.

\section{PALAVRAS-CHAVE}

Paralisia facial. Nervo facial. Nervo hipoglosso. Transferência de nervos. Anastomose hipoglosso-facial.

\section{ABSTRACT}

Hemihypoglossal-facial neurorrhaphy after intratemporal dissection of facial nerve: retrospective analysis of 13 patients

Background: The hypoglossal-facial neurorrhaphy has been an effective surgical procedure for reanimation of paralyzed facial muscles after an irreparable injury of the proximal facial nerve. In spite of good results obtained through this technique, the tongue dysfunction after total section of hypoglossal nerve may be quite disturbing. To avoid this complication some authors have been using a hemyhypoglossal-facial neurorrhaphy. This technique involves mobilization of the intratemporal portion of facial nerve with its proximal section allowing caudal dislocation and suture without tension with half of the sectioned hypoglossal nerve. Objective: To evaluate retrospectively the results of this technique in 13 patients. Method: The outcome was evaluated by grading the facial paralysis by House-Brackmann scale. Results: By the time of surgery the average age was 42.2 years and the delay between nerve injury and nerve transfer was 6.8 months. All patients had complete facial paralysis previous to surgery. In $70 \%$ of patients there was a good return of facial nerve function, improved from grade VI to grade II or III. There was no subjective tongue dysfunction. A significant correlation was identified between age and final outcome $(p<0.05)$ and between surgery delay and results $(p<0.05)$. Conclusions: Good results in facial reanimation can be obtained with the hemyhypolglossal-facial neurorrhaphy with low morbidity. We recommended that this technique should be applied as soon as possible after confirmed facial nerve injury.

\section{KEY-WORDS}

Facial paralysis. Facial nerve. Hypoglossal nerve. Nerve transfer. Hypoglossal-facial anastomosis.

1 Grupo de Cirurgia do Sistema Nervoso Periférico, Disciplina de Neurocirurgia do Hospital das Clínicas da Faculdade de Medicina da Universidade de São Paulo. São Paulo, SP.

2 Grupo de Cirurgia do Sistema Nervoso Periférico, Serviço de Neurocirurgia do Hospital Santa Marcelina. São Paulo, SP. 


\section{Introdução}

A neurorrafia hipoglosso-facial (NHF) é um método eficiente para a reinervação dos músculos da face, sendo indicada quando há lesão proximal e irreversível do nervo facial ${ }^{1,2,22,24}$. Essa situação ocorre principalmente após a exérese de neoplasias da região do ângulo pontocerebelar, mas outras condições como a lesão traumática intratemporal do nervo podem também resultar na impossibilidade do reparo direto deste ${ }^{24}$. A primeira NHF foi realizada por Korte em Berlim, em 1901 (citado por Pitty) ${ }^{24}$. Nessa técnica clássica, ainda amplamente utilizada, o nervo hipoglosso é identificado, seccionado, deslocado cranialmente e suturado de forma direta ao nervo facial, dissecado na região cervical alta. Em virtude da secção completa do nervo hipoglosso, ocorre uma inevitável atrofia da hemilíngua.

Para evitar a possibilidade da disfunção da hemilíngua, diversas técnicas têm sido utilizadas ${ }^{3,8,9,22,28}$. Em uma dessas técnicas, o nervo facial intratemporal é dissecado, seccionado e deslocado caudalmente, o que permite a sua sutura sem tensão com metade do nervo hipoglosso, reduzindo a possibilidade de disfunção da língua.

Neste estudo, apresentamos os resultados da técnica de neurorrafia hemiipoglosso-facial após transposição da porção intratemporal do nervo facial (NIF) utilizada em 13 pacientes com preservação da função da hemilíngua.

\section{Casuística e métodos}

Avaliaram-se, retrospectivamente, os resultados da NIF em 13 pacientes operados no Serviço de Neurocirurgia do Hospital Santa Marcelina, de São Paulo (SP), e na Disciplina de Neurocirurgia do Hospital das Clínicas da Faculdade de Medicina da Universidade de São Paulo, no período de fevereiro de 2003 a julho de 2006. No período considerado, a técnica foi aplicada em 17 pacientes. Em quatro pacientes, não foi possível avaliar os resultados em virtude da falta de seguimento.

A cirurgia foi realizada quando havia informação sobre uma lesão irreversível do nervo facial durante a exérese de um tumor na região do ângulo pontocerebelar, ou após o período de 8 meses de uma lesão fechada do nervo, sem que houvesse recuperação clínica ou eletroneuromiográfica.

A avaliação pré-operatória dos pacientes baseou-se na classificação de House-Brackmann (HB), que gradua a paralisia facial de I a VI, sendo o grau VI relacionado a uma lesão completa, com comprometimento severo do movimento da hemiface ${ }^{15}$.
A técnica cirúrgica utilizada foi semelhante às descritas previamente por Atlas e Lowinger ${ }^{3}$ e Sawamura e $\mathrm{Abe}^{28}$. Com o paciente sob anestesia geral, a cabeça é posicionada após a sua rotação contralateral à lesão e discreta flexão do pescoço. Realiza-se incisão linear retro-auricular, iniciando-se a $4 \mathrm{~cm}$ proximalmente à extremidade da mastóide $\mathrm{e}$ estendendo-se por cerca de $4 \mathrm{~cm}$ a $6 \mathrm{~cm}$, obliquamente, na região cervical lateral até um ponto situado a $2 \mathrm{~cm}$ posterior ao ângulo da mandíbula. Após a dissecção do tecido subcutâneo, a fáscia cervical é aberta longitudinalmente em relação à incisão cutânea com o músculo platisma. O músculo esternocleidomastóide é desinserido da ponta da mastóide, permitindo a exposição dessa estrutura. Com auxílio de magnificação por lupa, o nervo facial é localizado na saída do forâmen estilo-mastóideo, no espaço entre a ponta da mastóide e o processo estilóide, identificado por meio de palpação digital. A ponta da mastóide é ressecada com broca pneumática de alta rotação, permitindo melhor visibilização do nervo facial. A dissecção do nervo facial prossegue cranialmente no interior da mastóide, com o auxílio de uma broca de diamante. O nervo é dissecado no seu canal até a altura do joelho externo, no limite proximal de sua porção descendente (Figura 1). Todo o tecido conjuntivo adjacente ao nervo é preservado com o objetivo de manter a sua irrigação, originada preferencialmente da artéria estilomastóidea. O nervo facial é seccionado proximalmente e deslocado para a região cervical após a secção do nervo corda do tímpano (Figura 2). A cabeça posterior do músculo digástrico é retraída inferiormente, permitindo o acesso a veia jugular, artéria carótida e nervo hipoglosso. A identificação do nervo é possível por meio de sua relação com a artéria carótida, situada posteriormente a este. $\mathrm{O}$ nervo hipoglosso é dissecado proximalmente até a extremidade seccionada do nervo facial, permitindo o planejamento de uma sutura sem tensão. O nervo hipoglosso é seccionado transversalmente até a metade do seu diâmetro, permitindo a sutura entre essa estrutura e toda a secção transversa do nervo facial com 4 a 5 pontos de náilon monofilamentar 10-0 (Figura 3). Uma a duas suturas adicionais são realizadas entre o tecido conjuntivo do nervo facial e o epineuro do nervo hipoglosso, a cerca de $5 \mathrm{~mm}$ da neurorrafia, com o objetivo de se atingir um alinhamento mais eficiente dos dois cotos e reduzir uma eventual tensão entre as duas estruturas. Ao final do procedimento, a cavidade da mastóide é obliterada com um fragmento de músculo. Duas a três semanas após a cirurgia, os pacientes são encaminhados à fisioterapia para reabilitação.

Após a cirurgia, os pacientes foram questionados quanto à ocorrência de possíveis alterações relacionadas a fala e deglutição. Ao final do período de seguimento, realizou-se uma nova avaliação da integridade muscular facial mediante a quantificação do grau de paralisia pela escala de House-Brackmann. 


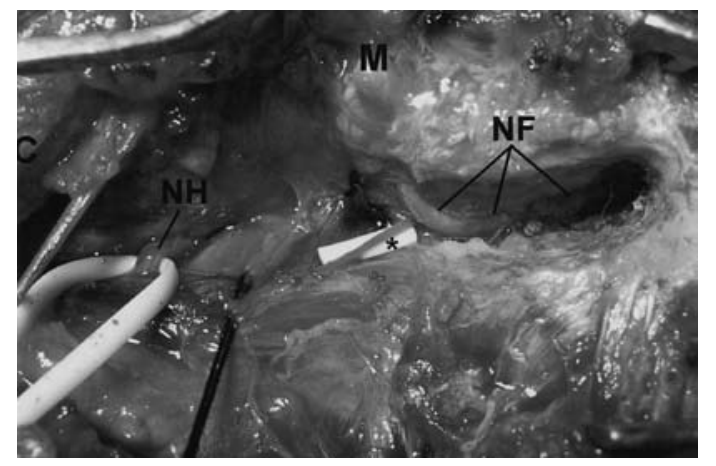

Figura 1 - Fotografia cirúrgica, lado esquerdo, evidenciando o nervo facial (NF) isolado após mastoidectomia parcial.

C: caudal; M: medial; NH: nervo hipoglosso; *ramo do nervo facial para o músculo digástrico.

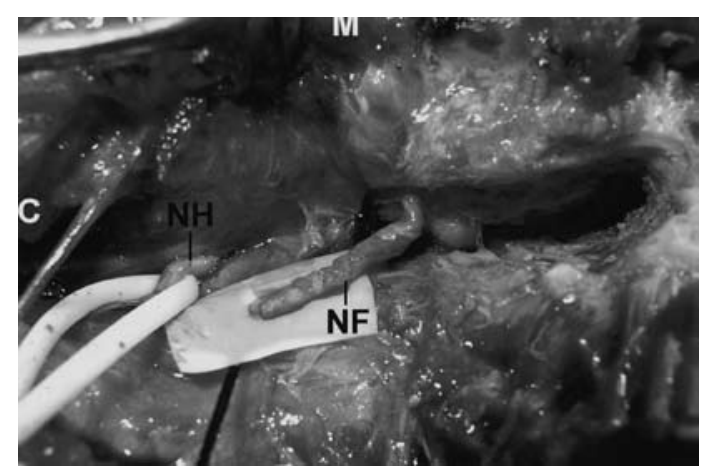

Figura 2-Fotografia cirúrgica, lado esquerdo, demonstrando o nervo facial (NF) seccionado e deslocado caudalmente na direção do nervo hipoglosso (NH). C: caudal; M: medial.

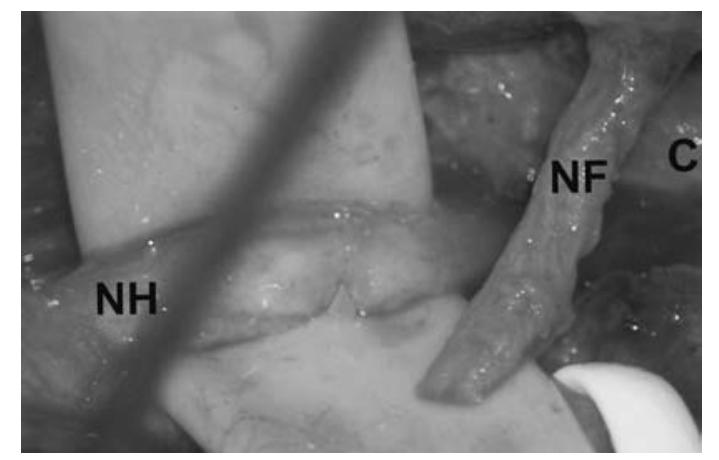

Figura 3-Fotografia cirúrgica, lado esquerdo, evidenciando o nervo facial (NF) deslocado para o local da neurorrafia. Note a secção parcial do nervo hipoglosso (NH). C: cranial; M: medial.

Avaliou-se a correlação entre a idade e o tempo desde a lesão do nervo facial até a cirurgia e os resultados após o período de seguimento. Realizou-se análise estatística com o uso do programa SPSS versão 14.0 para Windows (SPSS Inc., Chicago, IL, EUA). Após a realização do teste de Kolmogorov-Smirnov para avaliação da normalidade da amostra, obteve-se a correlação entre os fatores considerados e o resultado da cirurgia por meio da mensuração do coeficiente de correlação linear de Spearman (rho). As correlações foram consideradas significativas se os valores de $p$ fossem inferiores a 0,05 .

\section{Resultados}

A exérese de neoplasias da região do ângulo pontocerebelar foi a causa mais comum de lesão do nervo facial, responsável por $92,3 \%$ dos casos. Em somente um caso, a lesão do nervo foi secundária à fratura da base do crânio. A idade média dos pacientes no momento da cirurgia foi de 42,2 anos (intervalo de 23 a 62 anos). Todos os pacientes apresentavam comprometimento severo do nervo facial, sendo classificados como grau VI na classificação de HB. Realizou-se a cirurgia no período médio de 6,8 meses (intervalo de 1 a 15 meses) após a lesão do nervo facial. O seguimento médio foi de 20,8 meses (intervalo de 8 a 38 meses) após a cirurgia. Todos os pacientes apresentaram melhora de acordo com a classificação de HB: 3 pacientes foram classificados como grau II, 6 pacientes, como grau III, 3 pacientes, como grau IV e 1 paciente, como grau V (Figuras 4 e 5). Uma correlação positiva e significativa foi identificada entre a idade e o resultado final ( $\mathrm{rho}=0,58$, $\mathrm{p}<0,05)$ e o tempo decorrido entre a lesão e a cirurgia e o resultado desta $($ rho $=0,63, \mathrm{p}<0,05)$ (Figura 6). Apenas um paciente apresentou parestesia na hemilíngua, com resolução espontânea, e nenhum apresentou alteração relacionada a fala, mastigação ou deglutição. A atrofia da hemilíngua ocorreu em apenas dois casos $(15,4 \%)$, sendo considerada leve (restrita a $25 \%$ da hemilíngua) em um paciente e moderada (atingindo $25 \%$ a $50 \%$ da hemilíngua) em outro. Um paciente apresentou infecção superficial da ferida operatória com boa evolução após antibioticoterapia via oral. Nenhum paciente apresentou fístula de líquido cefalorraquidiano.

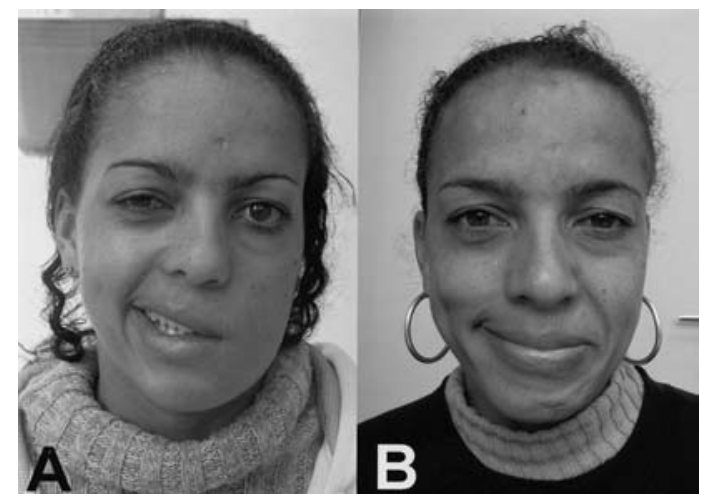

Figura 4-Fotografia de paciente de 27 anos antes $(A)$ e 15 meses após a neurorrafia hemi-ipoglossofacial (B), resultando em significativa melhora da simetria facial. 


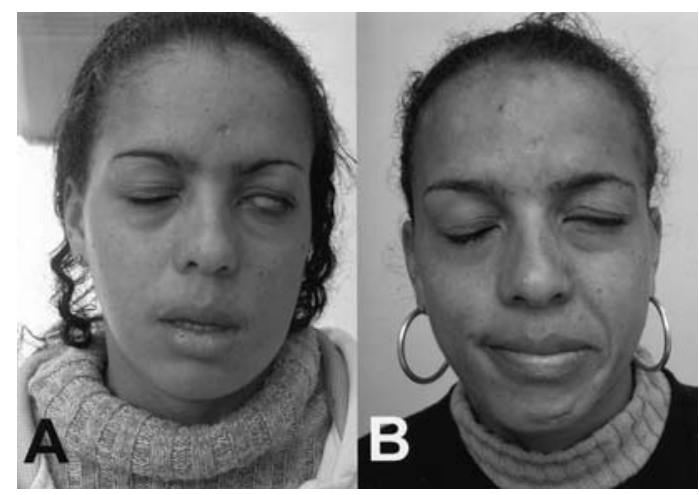

Figura 5-Fotografia de paciente de 27 anos antes $(A) e$ 15 meses após a neurorrafia hemi-hipoglossofacial (B), demonstrando restituição da oclusão palpebral.

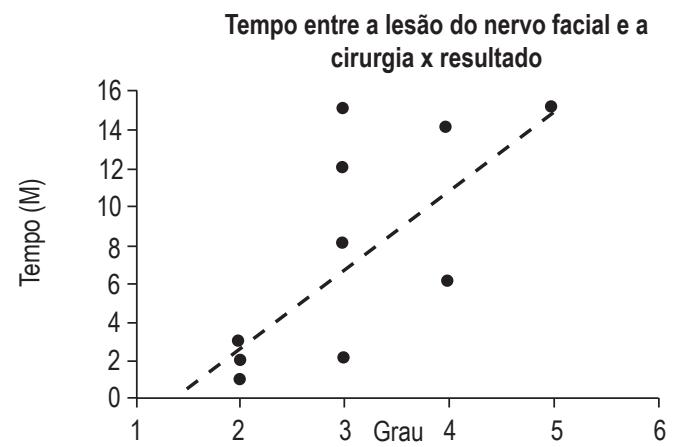

Figura 6-Gráfico demonstrando a relação entre os valores da graduação de House-Brackmann e o tempo entre a lesão do nervo facial e a cirurgia. M: meses.

\section{Discussão}

Apesar dos bons resultados observados com a técnica clássica da NHF, a hemiatrofia da língua é freqüente após a secção do nervo hipoglosso ${ }^{23}$. Em até $45 \%$ dos casos, a disfunção resultante da secção do hipoglosso da hemilíngua associa-se a algum grau de comprometimento da mastigação, da fala e da salivação, principalmente em pacientes portadores de disfunções de outros nervos cranianos, o que não é infreqüente em portadores de neoplasias da região do ângulo pontocerebelar ${ }^{23,24}$. A maioria dos autores contra-indica a técnica na presença de disfunção de outros nervos cranianos.

Para evitar ou reduzir as conseqüências da disfunção da hemilíngua, propôs-se uma série de variações da NHF, a maioria delas centrada na manipulação do nervo hipoglosso. Numa das primeiras modificações da técnica clássica, o ramo descendente do nervo hipoglosso é seccionado e suturado ao coto distal desse nervo, com resultados insatisfatórios em relação à manutenção do trofismo da hemilíngua ${ }^{4,524,32}$. Considera-se que a desproporção de fibras entre o ramo descendente e o nervo hipoglosso seja a principal causa desses resultados ${ }^{14,24}$. Em outra variação técnica, o nervo hipoglosso é dividido longitudinalmente ao longo do seu maior eixo e seccionado parcialmente, possibilitando o deslocamento dessa parte do nervo para se realizar uma sutura direta com o nervo facial, seccionado próximo à sua origem no forâmen estilomastóideo ${ }^{1,7}$. Apesar de preservar metade do nervo, observou-se atrofia leve a moderada em todos os pacientes ${ }^{1}$. Como o nervo hipoglosso é monofascicular, a divisão longitudinal deste por uma longa extensão leva à lesão dos axônios que cruzam o fascículo em vários pontos da área de secção ${ }^{28}$. Mais recentemente, tem-se utilizado a secção transversa de parte do nervo hipoglosso para evitar as possíveis complicações de uma secção completa. De acordo com estudos morfométricos e considerando os resultados de estudos experimentais, os axônios de metade do nervo hipoglosso seriam suficientes para promover a reinervação da hemiface ${ }^{2,17,19,31}$. Algumas variações técnicas diferem na forma de se realizar a conexão entre parte do nervo hipoglosso e o nervo facial. Na técnica proposta por May e cols. ${ }^{22}$, obtém-se essa conexão por meio da interposição de um enxerto entre as duas estruturas. Apesar dos resultados satisfatórios em relação à manutenção da função da hemilíngua, essa técnica tem a desvantagem da morbidade relacionada à retirada do enxerto e a necessidade de o axônio em regeneração ultrapassar duas suturas ${ }^{9,14,20,22}$. A fim de se obter uma extensão de nervo facial suficiente para a realização de uma sutura única com parte do nervo hipoglosso, Sawamura e $\mathrm{Abe}^{28}$, em 1997, descreveram uma nova técnica de neurorrafia hipoglosso-facial em quatro pacientes, com preservação da função da hemilíngua. Na técnica descrita pelos autores, a exposição do nervo facial no seu canal permite que este seja deslocado após a sua secção proximal até o local da neurorrafia com metade do nervo hipoglosso, possibilitando a realização de uma sutura sem tensão e com manutenção da função da hemilíngua ${ }^{2,3,9,28}$.

Diversos fatores influenciam os resultados após cirurgia de nervo. A idade é geralmente considerada um fator que influencia os resultados finais após a neurorrafia hipoglosso-facial, pois em geral quanto mais jovem o paciente, melhores os resultados, relação também observada na presente série ${ }^{13,20,21,24}$. A influência do tempo entre a lesão do nervo facial e a cirurgia e os resultados finais na neurorrafia hipoglosso-facial ainda é motivo de controvérsia na literatura. Geralmente os melhores resultados relacionam-se à precocidade da cirurgia, mas resultados satisfatórios foram obtidos em neurorrafias realizadas mais de dois anos após a lesão do nervo facial ${ }^{5,6,10,13,24,27}$. Adicionalmente, alguns autores não identificaram nenhuma relação entre a demora na realização da cirurgia e o resultado final ${ }^{12,16,18,26,28,29}$. 
Na presente série, observou-se uma correlação positiva e significativa entre a precocidade da cirurgia e os melhores resultados. A justificativa para a ocorrência de bons resultados mesmo após demora significativa da cirurgia é a distância curta entre o nervo e os músculos faciais, reduzindo a distância percorrida pelos axônios em regeneração. Além disso, o crescimento de axônios colaterais a partir da hemiface contralateral, fenômeno observado em estudos clínicos e experimentais, reduz a possibilidade da ocorrência de alterações tróficas irreversíveis na musculatura desnervada ${ }^{11,30}$.

Poucos trabalhos têm sido publicados avaliando a técnica descrita e são restritos a estudos com um número reduzido de $\operatorname{casos}^{3,8,9,25,28}$. Os resultados da presente casuística mostram que a técnica adotada apresenta resultados comparáveis com os das que utilizam a técnica clássica, com a vantagem de reduzir a incidência de complicações relacionadas à atrofia da hemilíngua ${ }^{8,9,18,27}$. Em virtude desse fato, a NIF pode ser considerada opção terapêutica mesmo nos pacientes portadores de outras lesões de nervos cranianos baixos e em pacientes com neurofibromatose tipo II. A morbidade observada foi reduzida, mas recomenda-se que a cavidade resultante da mastoidectomia seja obliterada para evitar a ocorrência de fístula de líquido cefalorraquidiano; essa recomendação foi seguida rotineiramente nos casos por nós operados. É importante ressaltar que a cirurgia exige o conhecimento anatômico do nervo facial no seu trajeto intratemporal, sendo mais trabalhosa e mais demorada em relação à técnica clássica, pois a dissecção e mobilização do nervo devem ser cuidadosas para evitar possíveis lesões. Mesmo assim, consideramos que o acesso intratemporal do nervo facial deva ser um procedimento rotineiro para os cirurgiões que manipulam o nervo na região cervical alta. Em alguns casos, o nervo facial apresenta um calibre reduzido em razão de um longo período decorrido entre a lesão do nervo e a cirurgia e a sua identificação pode exigir a aplicação desse tipo de técnica.

\section{Conclusão}

A neurorrafia hemiipoglosso-facial é uma técnica adequada para a reinervação da hemiface após uma lesão proximal do nervo facial, permitindo a manutenção da função da hemilíngua, com baixa morbidade. Para que os resultados sejam otimizados, recomendamos que a cirurgia seja realizada o mais precocemente possível após a lesão do nervo facial.

\section{Referências}

1. ARAI H, SATO K, YANAI A: Hemihypoglossal-facial nerve anastomosis in treating unilateral facial palsy after acoustic neurinoma resection. J Neurosurg 82:51-4, 1995.

2. ASAOKAK, SAWAMURA Y, NAGASHIMAM, FUKUSHIMA T: Surgical anatomy for direct hypoglossal-facial nerve sideto-end "anastomosis". J Neurosurg 91:268-75, 1999.

3. ATLAS MD, LOWINGER DSG: Anew technique for hypoglossal-facial nerve repair. Laryngoscope 107:984-91, 1997.

4. CHANG CGS, SHEN AL: Hypoglossal anastomosis after resection of acoustic neuroma. Surg Neurol 21:282-6, 1984.

5. CONLEY J, BARKER DC: Hypoglossal-facial nerve anastomosis for innervation of the paralysed face. Plast Reconstr Surg 63:63, 1979.

6. CONSTANTINIDIS J, AKBARIAN A, STEINHART H, IRO H, MAUTES A: Effects of immediate and delayed facial-facial nerve suture on rat facial muscle. Acta Otolaryngol 123:9981003, 2003.

7. CUSIMANO MD, SEKHAR L: Partial hypoglossal to facial nerve anastomosis for reinnervation of the paralyzed face in patients with lower cranial nerve palsies: technical note. Neurosurgery 35:532-34, 1994.

8. DARROUZET V, GUERIN J, BÉBÉAR J: New technique of side-to-end hypoglossal-facial nerve attachment with translocation of the infratemporal facial nerve. J Neurosurg 90:27-34, 1999

9. DARROUZET V, DUTKIEWICZ J, CHAMBRIN A, STOLL D, BEBEAR JP: L'anastomose hypoglosso-faciale: resultats et evolution technique vers l'anastomose latero-terminale avec deroutment du nerf facial intra-temporal (technique de May modifiee). Rev Laryngol Otol Rhinol 118:203-10, 1997.

10. GAVRON JP, CLEMIS JD: Hypoglossal-facial nerve anastomosis: a review of forty cases caused by facial nerve injuries in the posterior fossa. Laryngoscope 94:1447-50, 1984.

11. GILHUIS HJ, BEURKENS CH, DE VRIES J, MARRES HA, HARTMAN EH, ZWARTS MJ: Contralateral reinnervation of midline muscles in nonidiopathic facial palsy. J Clin Neurophysiol 20:151-4, 2003.

12. GUNTINAS-LICHIUS O, ANGELOV DN: Delayed hypoglossal-facial nerve suture after predegeneration of the peripheral facial nerve stump improves the innervation of mimetic musculature by hypoglossal motoneurons. J Comp Neurol 387:234-42, 1997.

13. GUNTINAS-LICHIUS O, STREPPEL M, STENNERT E: Postoperative functional evaluation of different reanimation techniques for facial nerve repair. Am J Surg 191:61-7, 2006.

14. HAMMERSCHLAG PE, BRUDNY J, CUSUMANO R et al.: Hypoglossal-facial nerve anastomosis and electromyographic feedback rehabilitation. Laryngoscope 97:705-9, 1987.

15. HOUSE JW, BRACKMANN DE: Facial nerve grading system. Otolaryngol Head Neck Surg 93:146-7, 1985.

16. HWANG K, KIM SG, KIM DJ: Facial-hypoglossal nerve anastomosis using laser nerve welding. J Craniofac Surg 17:687-91, 2006

17. KALANTARIAN B, RICE DC, TIANGCO DA et al.: Gains and losses of the XII-VII component of the "baby-sitter" procedure: a morphometric analysis. J Reconstr Microsurg 14:459-71, 1998.

18. KUNIHIRO T, KANZAKI J, YOSHIHARA S, SATOH Y, SATOHA: Hypoglossal-facial nerve anastomosis after acoustic 
neuroma resection: influence of the time anastomosis on recovery of facial movement. J Otorhinolaryngol Relat Spec 58:32-5, 1996.

19. MACKINNON SE, DELLON AL: Fascicular patterns of the hypoglossal nerve. J Reconstr Microsurg 11:195-8, 1995.

20. MAGLIULO G, D'AMICO R, FORINO M: Results and complications of facial reanimation following cerebellopontine angle surgery. Eur Arch Otorhinolaryngol 258:45-8, 2001.

21. MALIKTH, KELLY G, AHMEDA, SAEED SR, RAMSDEN RT: A comparison of surgical techniques used in dynamic reanimation of the paralyzed face. Otol Neurotol 26: 284-91, 2005.

22. MAY M, SOBOL SM, MESTER SJ: Hypoglossal-facial nerve interpositional-jump graft for facial reanimation without tongue atrophy. Otolaryngol Head Neck Surg 104:818-25, 1991.

23. PASCUAL LH, ARRIBAS MA: Résultats des anastomoses hypoglosso-faciale et hypoglosso-hypoglosse. Neurochirurgie 42:209-15, 1996

24. PITTY LF, TATOR CH: Hypoglossal-facial nerve anastomosis for facial nerve palsy following surgery for cerebellopontine angle tumors. J Neurosurg 77:724-31, 1992.

25. REBOL J, MILOJKOVIC V, DIDANOVIC V: Side-to-end hypoglossal-facial anastomosis via transposition of the intratemporal facial nerve. Acta Neurochir (Wien) 148: 653-7, 2006.

26. ROLAND JR JT, LIN K, KLAUSNER LM, MILLER PJ: Direct facial-to-hypoglossal neurorrhaphy with parotid release. Skull Base 16:101-107, 2006.

27. SAMII M, MATTHIES C: Indication, technique and results of facial nerve reconstruction. Acta Neurochir (Wien) 130: 125-39, 1994
28. SAWAMURA Y, ABE H: Hypoglossal-facial nerve side-to-end anastomosis for preservation of hypoglossal function: results of delayed treatment with a new technique. J Neurosurg 86:203-6, 1997

29. SOOD S, ANTHONY R, HOMER JJ, VAN HILLE P, FENWICK JD: Hypoglossal-facial nerve anastomosis: assessment of clinical results and patient benefit for facial nerve palsy following acoustic neuroma excision. Clin Otol Allied Sciences 25:219-26, 2000.

30. TANKERE F, BERNAT I, VITTE E, LAMAS G, BOUCHE P, FOURNIER E, SOUDANT J, WILLER JC: Hypoglossalfacial nerve anastomosis. Dynamic insight into the crossinnervation phenomenon. Neurology 61:693-5, 2003.

31. THURNER KH, EGG G, SPOENDLIN H, SCHROTT-FISCHER A: A quantitative study of nerve fibers in the human facial nerve. Eur Arch Otorhinolaryngol 250:161-7, 1993.

32. VACHER C, DAUGE MC: Morphometric study of the cervical course of the hypoglossal nerve and its application to hypoglossal facial anastomosis. Surg Radiol Anat 26:86-90, 2004.

Original recebido em julho de 2007

Aceito para publicação em dezembro de 2007

\section{Endereço para correspondência}

Roberto Sergio Martins

Rua Maestro Cardim, 592, cj. 1.101

01323-001 - São Paulo, SP

E-mail: robar@ig.com.br 